\title{
Improving Power Quality and Mitigation of Harmonic Distortion Impact at Photovoltaic Electric Vehicle Charging System
}

\author{
Adel Elgammal, Curtis Boodoo
}

\begin{abstract}
This article offers a clear and realistic design for an active power filter to increase reliability and power quality of the photovoltaic charging system and a high-penetration electric vehicle distribution system. The MOPSO algorithm is used as the basis for problems with optimization and filter tuning. A typical regular load curve is used to model the warped power grid over a 24-hour cycle to estimate the total harmonic distortion (THD). For structures with high penetration of electric cars, the probability of minimizing THD (for example to five percent) is explored via optimum capacity active shunt filters and shunt capacitors. To maximize general performance of the charging system, the switching systems are re-scheduled. Moreover, to increase the current control accuracy of shunt active filter, the fuzzy logic controller is utilized. The major drawback to new system is that it would have unrestricted billing for entire day to cope with voltage interruption. In MATLAB / SIMULINK, detailed machine setup and control algorithm experiments are simulated. The simulation findings confirm the efficiency and viability of projected shunt active filter to enhance voltage profile and track power performance of photovoltaic charging system.
\end{abstract}

Index Terms - Harmonics, Power Quality, Total Harmonic Distortion, Photovoltaic (PV) charging system, Plug-In Electric Vehicles, Shunt Active Power Filter, MOPSO.

\section{INTRODUCTION}

Plug-in electric vehicles are becoming more popular, providing more efficient generators, grid surplus energy storage, and vehicle-to-grid capacity to enable peak-period grid support and transition to cleaner technology. There is Reduction of greenhouse gas emissions as a result of federal business restrictions and public sentiment [1]. However, the rapid rise in EV and the non-linearity of the power transfer of the charging station has improved the quality of voltage and harmonic distortion problems affecting the output of smart grid electrical transmission networks and interconnected renewable energy sources (solar and wind). In the delivery feeders, serious power quality issues have occurred, such as flicker, resonance and electrical equipment disturbance, transmission line losses and heating, engine vibrations and noise, breakdown and metering/sensitive equipment faults [2]. The results of the sinusoidal and non-sinusoidal currents of the charging station have been found to greatly increase the dissipation of harmonic and fundamental losses. An active filter must be mounted parallel to charging station. The harmonics cancel to offset the unexpected results of temperature increase and further damages from existing

Published on January 20, 2021.

Adel Elgammal, The University of Trinidad and Tobago UTT, Utilities Engineering, Point Lisas Campus, Trinidad and Tobago.

(corresponding e-mail: adel_elgammal2000@yahoo.com) harmonics inserted through charging stations [3].

PEVs must be charged in public or private parking lots, at power charging points or at the user's home. This would play a significant role in the recent generation of smart grids planned to update the century old distribution system architecture for potential electricity needs [4]. It is expected that charging stations will be situated in suburban and light industrial areas. Distribution systems can face stress due to sudden power demand spikes because of their great ratings for fast charging PEVs in little bursts [5]. Moreover, EV has also been affected by the unstable price of crude oil to become a viable substitute. The market for EV has indirectly increased the number of installations of charging stations in the delivery system [6]. However, where a significant number of unplanned charging stations are installed, power losses can increase. In addition, harmonic distortion due to electronic control systems transferring alternating current to direct current at charging stations would also be added [7], [8]. This harmonic distortion will have a detrimental effect, such as lower efficiency, lower power factor, higher insulation temperature voltage, shorter insulation life and higher heating losses [9], [10]. One field being studied is the total harmonic distortion, reactive power distribution and simultaneous control of voltage profile. Broad studies have been undertaken to implement client requirement side control into smart grids to decrease peak demand and increase the load profile of the system [11], [12].

Moreover, two kinds of harmonics, which are voltage and current harmonics, may be mitigated in the transmission network. On the bus, harmonic voltage is determined, while on the wires and cables, harmonic current is measured. A regular element used to block certain harmonics is passive filters. Passive filters can give way to harmonic currents of low impedance and thus inhibit flow through systems [13]. There are several publications that use power grid passive filters to remove harmonics utilizing filter devices, and single tuned filters are the most common [14]. Single tuned filters are intended to remove or minimize a particular frequency depending on architectural capacity, capacitance and gain of inductance intensity from the device [15]. Due to photovoltaic link, a hosting capability determination for a skewed delivery scheme has been proposed. Passive filters are utilized to optimize the harmonic-constrained hosting space, which later raises harmonic filtering, power factor, and voltage [16]. To decrease the harmonic voltage compensator in a harmonic voltage compensator, an Asymmetric

Curtis Boodoo, The University of Trinidad and Tobago UTT, Utilities Engineering, Point Lisas Campus, Trinidad and Tobago. 
Synchronous Reference Frame Control System has been proposed [17]. To decrease THD voltage, harmonic voltage compensator was used. Although numerous scientists used passive filters to alleviate harmonics. Very rare scientists, especially for charging stations, considered both current and voltage harmonics in their study. Initially, due to their low original price, fast setup and high performance, passive filters with tuned LC parts were often used to suppress harmonic current [18]. But, passive filters have certain disadvantages, for example fixed compensation, utility impedances, series and parallel load resonance [19]. The above passive filter drawbacks can be reducing via using active power filters for harmonic exclusion and reactive power advantage [20]. Active power filters can be categorized as a series or shunt owing to their device design. The mixture of series and shunt active control filters is dubbed combined power quality compensator. One of the most critical corrective measures to address current harmonic issues at the source is the SAPFF [21]. In relation to harmonic load current, shunt active power filter is linked to load in parallel and compensation current is generated. Multilevel NPC inverters have been increasingly popular in the active power filter application world in latest years. This is due to the advantages of these topologies, the dimension of filter elements required, the low $\mathrm{dv} / \mathrm{dt}$, the better efficiency of the system, the lower common mode voltages and less electromagnetic interference, along with small harmonic alteration of the emitted current and voltage [22]. The efficacy of active filter topologies depends primarily on the technique of producing reference current. Any lack of precision in reference currents contributes to improper compensation [23]. The different techniques were used to test the SAPF reference present in the literature. These may be categorized into frequency domain or time domain approaches [24]. Paralleled to frequency domain approaches, the time domain methods require smaller computations. One of the most commonly used methods is the $\mathrm{p} q$ theory, or traditional prompt reactive power theory [25]. However, idea of harmonic components using the $\mathrm{p}-\mathrm{q}$ theory depends largely on performance of the electrical power grid voltage. In SAPF systems, the voltage components are then centered on $\mathrm{p}-\mathrm{q}$ theory, and this is normally achieved using the phase locked loop method [26]. But, there are a number of transformations necessary for this theory. However, in balanced conditions, these changes also take the power grid into account, which is not real state of the electrical network. The suggested control technique used is constructed on a simplified form of the $\mathrm{p} \mathrm{q}$ theory in order to resolve this trouble, using two HSFs to explicitly extract the basic variable from electrical signals (current and voltage) in alpha- $\beta$ relationship frame. Rather than traditional harmonic extractions centered on high pass or low pass filters, LSF was utilized in this technique. Instead high-pass filter, good current SAPF controller is used, after efficient extraction of the reference current, to maintain the active power filter currents at the imposed reference value [27], [28]. In some books, several researchers use different control approaches, such as PI, PID, sliding-mode controllers, etc. The PI controller, however, requires a particular linear mathematical model that is difficult to achieve and does not provide reasonable output under various circumstances, such as parameter differences, unbalanced or skewed AC voltages, etc. Compared to the PI controller, the fuzzy logic controllers have benefits, for example: it is more stable than the PI controller, it can handle nonlinearity, it can operate with imprecise inputs, and it needs no specific mathematical model [29], [30].

Most researchers are currently focused on techniques for minimizing damages by organizing the charging of EVs. To coordinate the charging of EV by means of the Genetic Algorithm, an optimization algorithm was developed [31]. The charging strategy is constructed on optimal load patterns that take into account thermal line constraints, transformer loads, voltage limits and patterns of parking availability for EV charging-based reliability. The proposed plan is capable of reducing the expense of new electricity grid projects. In addition, the approach for arranging EVs in the distribution system was proposed with the use of bi-level hierarchical vehicle grid optimization. [32]. This arrangement includes exchanging information between EV aggregator and grid controllers, and on the basis of reduced transmission network losses, EV charging can then be planned. Afterward, with use of met heuristic methodology, particle swarm optimization, online overnight PEV collaboration has been suggested [33]. A low-priority and high-priority client that reflects user's need to be paid is part of this bundle. High priority customers can receive faster high tariff charging facilities and only by testing the power outage in the distribution grid can the low priority customer charge their vehicle. While several techniques to mitigate the impact of the EV have been adopted, the emphasis is more on mitigating the loss of control. However, when many charging stations are linked, the harmonic injection into the supply grid must also be taken into consideration, apart from power outage, to ensure that the system is able to perform efficiently.

This article explores the effect of the Plug In Electric Car charging stations on reliability and power operation of the transmission system. Due to their broad classification of acdc power conversion electronics, PEV charging stations are large non-linear loads. The resulting current harmonics will cause irregular activity in smart grids, such as additional losses and decreased performance. The stability, safety, performance and economy of recently built smart grids may be greatly influenced by this. The Shunt active power filter requires an energy supply to overcome current-based distortions, using the DC-DC boost converter photovoltaic array. The DC connection voltage and the reactive and active power transmitted from renewable energy sources to the grid are controlled by the shunt linked inverter with improved power efficiency. This device uses solar energy and hence saves energy, exchanges the load, and delivers the critical/sensitive load with an uninterruptible power supply via the photovoltaic array during the day and night. This paper models an optimized charging and battery storage device for electric vehicles that functions in the presence of volatile solar sources. This research focuses on efficient switching control of the active power filter to minimize harmonic distortion due to a PV charging station delivery network planned within 24 hours, with a 15 -minute interval dependent on consumer activity. MOPSO is used to find optimum switching synchronization by taking into account the different charging station patterns and harmonic effects, so that less harmonics are applied to medium voltage network and energy loss is thereby minimized. The MOPSO is 
therefore an effective harmonic mitigation tool and is helpful in mitigating the effect of vigorous construction of PV charging stations on the distribution network. The paper suggested an iterative MOPSO-based mitigation approach to boost the power efficiency of smart grids with high EV charging station penetration. Optimum switching control of the distribution system's power active filter will eliminate harmonic injection into the distribution network. The paper has shown that MOPSO can decide optimum switching with Fuzzy control to decrease harmonic distortion and apparent distribution system losses. The fuzzy logic controller is utilized to map compensation currents of shunt active power filter.

\section{DESCRIPTION OF PROPOSED SYSTEM}

In order for PEVs to be a realistic alternative to longdistance travel equivalent to conventional fast charge PEV petrol stations or fuel based automobiles, they will have to be deployed at several situations nearby the network. The proposed charging station for ten fast electric car chargers is presented in Fig 1. A step-down 20/0.4 kV V mains distribution transformer valued at $500 \mathrm{KVA}$ interconnects the planned charging station with the distribution network. The large voltage diode rectification device with the same rating assumes the transformation of AC power into dc power needed for battery charging. Up to 10 rapid charger modules are assumed to have a regular dc bus. The overall no of PEVs heading to the charging station is restricted to $10 \mathrm{PEVs}$ by contrast, which is equal to that of a traditional gasoline station. For $10 \mathrm{EV}$, the maximum overall power is $50 \mathrm{~kW}$. At the same charging station, there is a $50 \mathrm{~kW}$ PV power plant installed. The PV system should be equipped with a highfrequency switching charger, which makes it capable of compensating for high-order harmonics. This PV inverter is used to minimize the harmonics of the inclusion of these ten EVs.

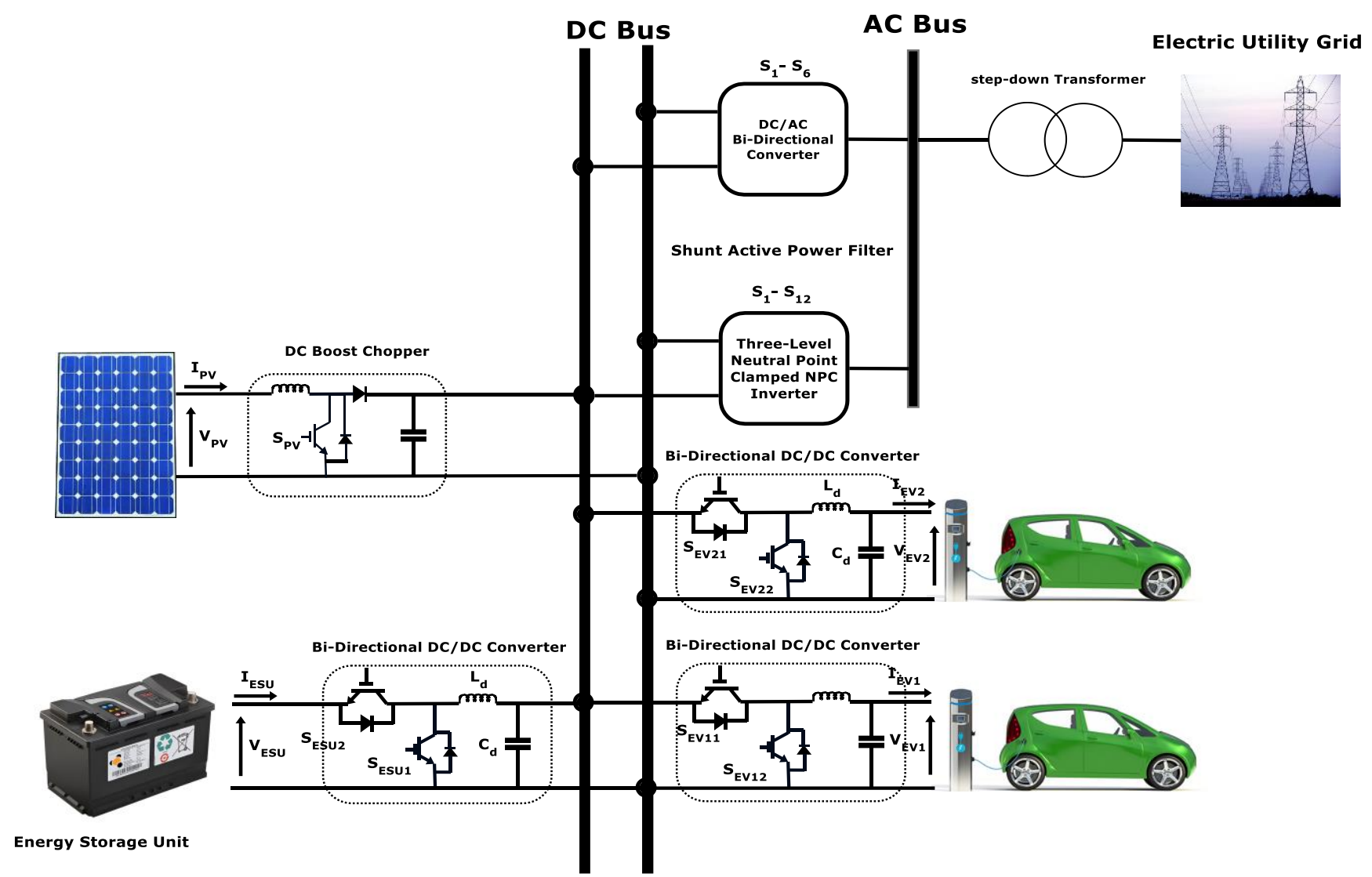

Fig. 1. The proposed Electric Motor Plug-In charging station with ten (10) fast electric vehicle chargers.

The reduction of current harmonics is accomplished by introducing into the shunt active power buffer the equal and opposite phases of the harmonic constituents of nonlinear load current at the point of the regular coupling. It thus renders current of source in phase with source voltage. The suggested configuration of the PV centered shunt active power filters, as seen in the figure. A DC/AC converter and bi-directional DC/DC converter comprised of two phases to charge the battery as a reversible chopper 2 . The three-level inverter of the Neutral Point Clamped NPC is connected to the main grid. Each leg consists of four controllable switches (S1-S4) for 3 levels Neutral Point Clamped NPC Inverter with two clamping diodes. If we consider that the two VDC1 and VDC2 capacitor voltages in the DC connection are equivalent, 3 voltage intensities (VDC/2, -VDC/2 and 0) are produced on $\mathrm{AC}$ terminal output of proposed inverter. The shunt active power filter is linked in conjunction with a nonlinear load to generate harmonic and reactive currents that overpower nonlinear loads to abandon harmonic currents. The projected structure includes a PV array with a DC DC boost converter and an MPPT feature to act as an inverter shunt source for DC voltage. Because of the fluctuating nature of solar energy produced, the electrical device operated through the PV array involves a DC-DC converter, 
triggered by abrupt changes in weather conditions that alter the degree of solar irradiation along with the operating temperature of the cell. Three modes of operation have been divided into the suggested PV-shunt active power filter procedure. These modes are of following three type.

I) Inverter mode: In this phase, during the available daytime or solar irradiation, the operation continues to operate. The projected PV Filter is used to serve as reactive and harmonic power compensator at this time and shares normal load. The PV array's unnecessary capacity charges the battery at the same time.

II) Compensator mode: The unit is only used to pay for voltage and current centered alterations and reactive power, while PV array does not receive any active power. The DCDC boost converter attached to the battery handles DC-link to efficiently deliver reimbursement continuity.

III) UPS mode: During the day and night, and throughout the voltage disruption time, PV-Filter guarantees the continuous supply of power to critical loads via the PV array and battery bank.
The key goals of this article are to preserve the DC connection voltage of parallel linked inverter clamped threelevel neutral point to deliver nonstop compensation, use green energy, share the load and deliver critical/sensitive load uninterrupted power supply. To raise the voltage and retain DC associated voltage constant, PV array is used to operate elevated step up DC DC boost converter. The PV array is connected to the boost converter during the daytime, for continuous gain, and shares the load with the transmission grid. The battery would serve as a DC source for the boost converter during the night period. This power is only used for compensation. After the device or the excess power produced from the PV array does not require compensation, it charges the battery. The proposed system also reduces the UPS and stabilizer criteria for specific devices. A low step up DC to DC converter associated with a feature named maximum power point monitoring is added between the PV array and battery to achieve optimum usage of the PV configuration. To validate the suggested procedure, the simulation and experimental findings are presented.

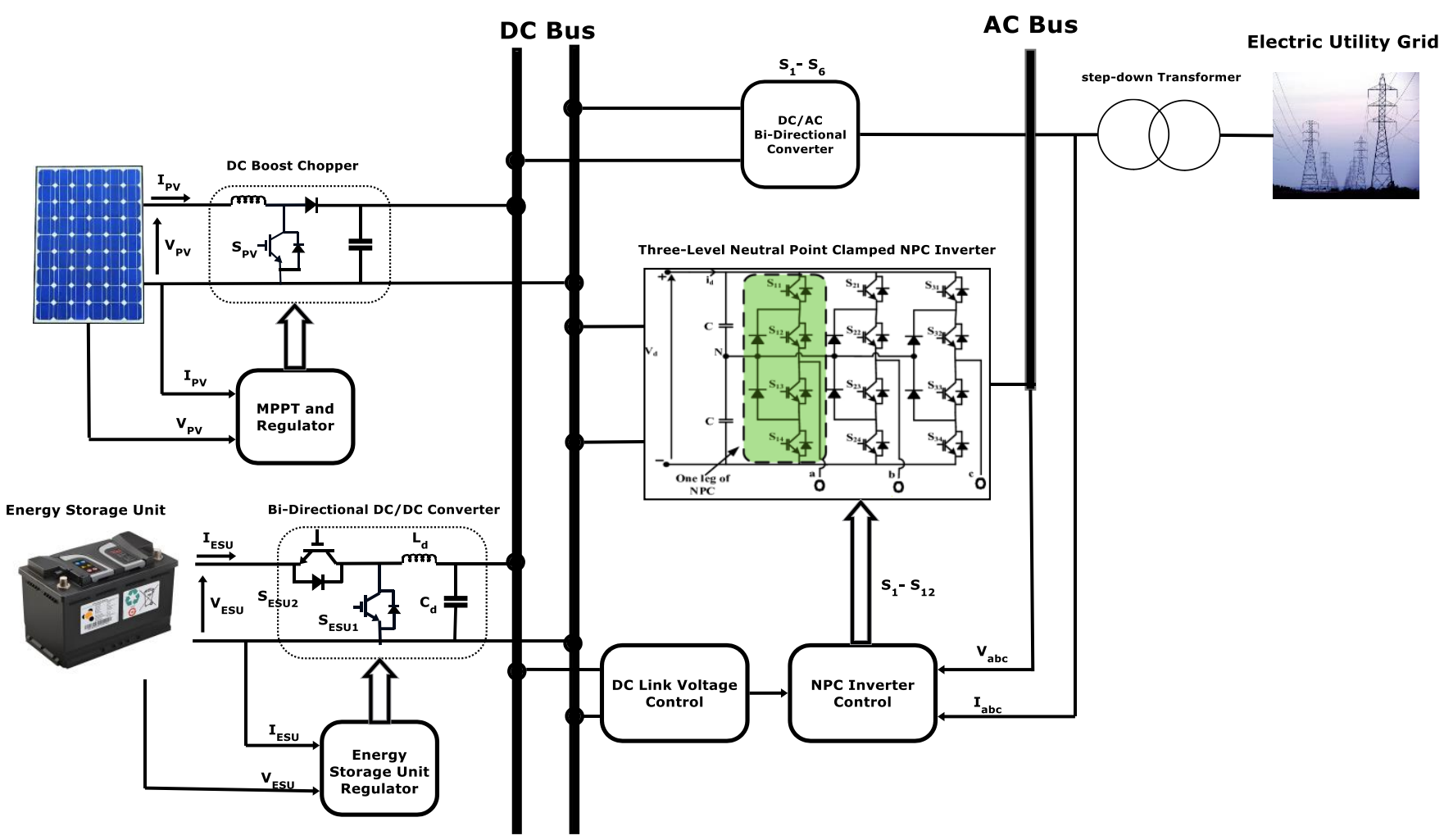

Fig. 2. The topology and control structure of the PV charging scheme.

In a simplified block diagram seen in Fig. 3, the main stages of recommended control system are illustrated. To control the active filter, a reference current was needed in order to supply a current opposite in the direction and equivalent to the amplitude of load current. By using the instantaneous reactive power principle in this control technique, the reference current signal is resultant from the measured quantities. The efficacy of active filter depends essentially on the modulation system and the current controller that has been introduced. Several modulation techniques for multilevel converters in three-phase system have been proposed. The methods of modulation are typically separated into two key groups. The first approach is based on comparators of hysteresis, and is characterized by a very basic and fast to execute, which also has the downside of uncontrollable switching frequency. The next approach is PWM, which requires a fixed switching frequency to work, such as sinusoidal PWM and space vector modulation. The carrier based PWM is used in this proposed method to produce the necessary exchanging signals of power switches, since it has the advantage of making the measurement process substantially easier and because of its operation at a fixed switching frequency. Subtracting the filter currents with the reference currents produces the switching pulses. The corresponding errors (e) and (de/dt) are submitted to the handler of fuzzy logic. The key goal of architecture of fuzzy logic controller is to decrease the flexibility of control system while ensuring a high degree of dynamic output and static 
processes with complicated modeling or unavailable parameters. It is the duty of the new control loop to control active power filter currents in projected control system circuit so that current is the same as the current relationship.

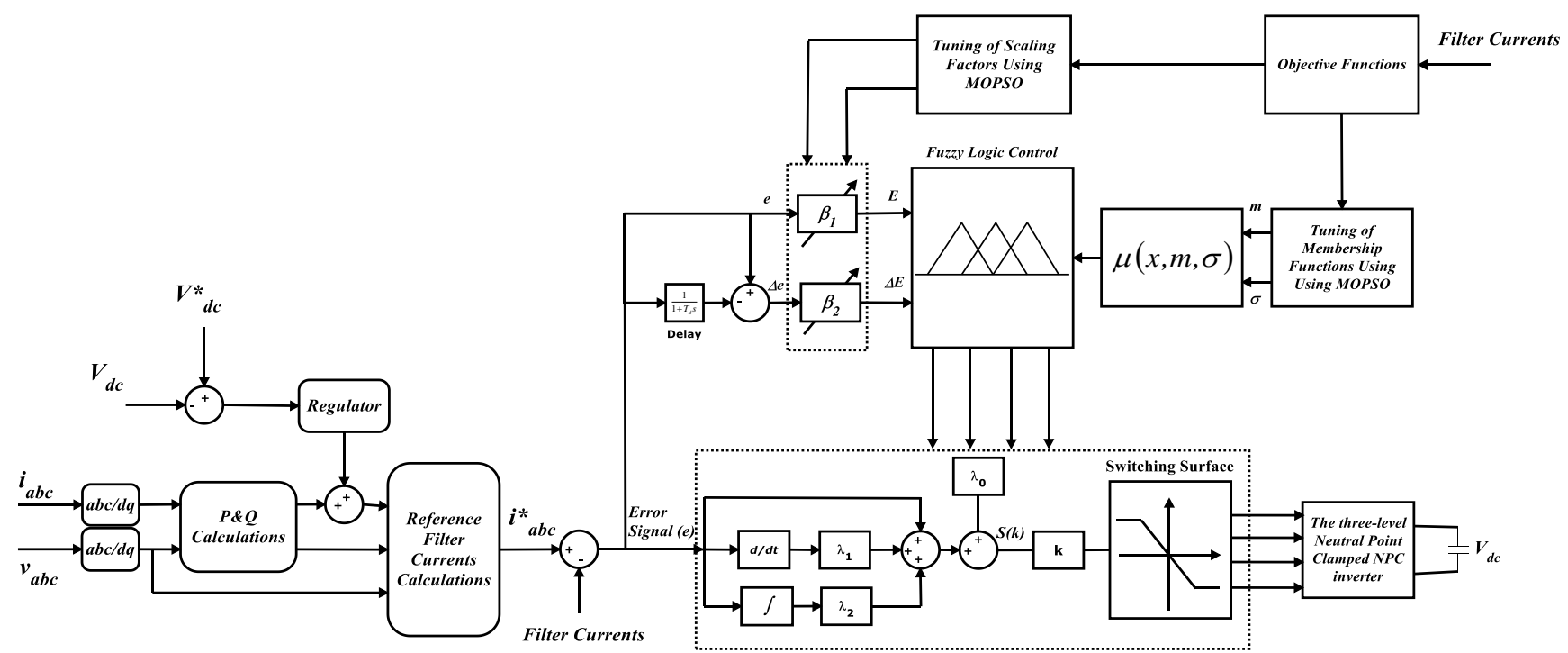

Fig. 3. The proposed Fuzzy logic controller scheme.

As PEV charging stations are relatively recent, it can be very difficult to forecast potential normal loading habits. It is fair to think, however, that the operation of PEV charging stations will follow figures similar to the actions of the vehicles' arrival and departure at conventional fuel stations. For a charging station, the presumed PEV charging operation and daily load curve are shown in Fig 4. The peaks in the morning and evening contribute to a rise in requirement for charging PEV before and after work. Like conventional fuel stations, two service bursts feature this load slope. When drivers board their vehicles in the morning to go to work, the first wave appears. The second peak refers to drivers who want their cars to top up after returning from work. This load curve is utilized to model various power intensities and demand patterns over a 24-hour cycle to quantify the impact on transformer efficiency.

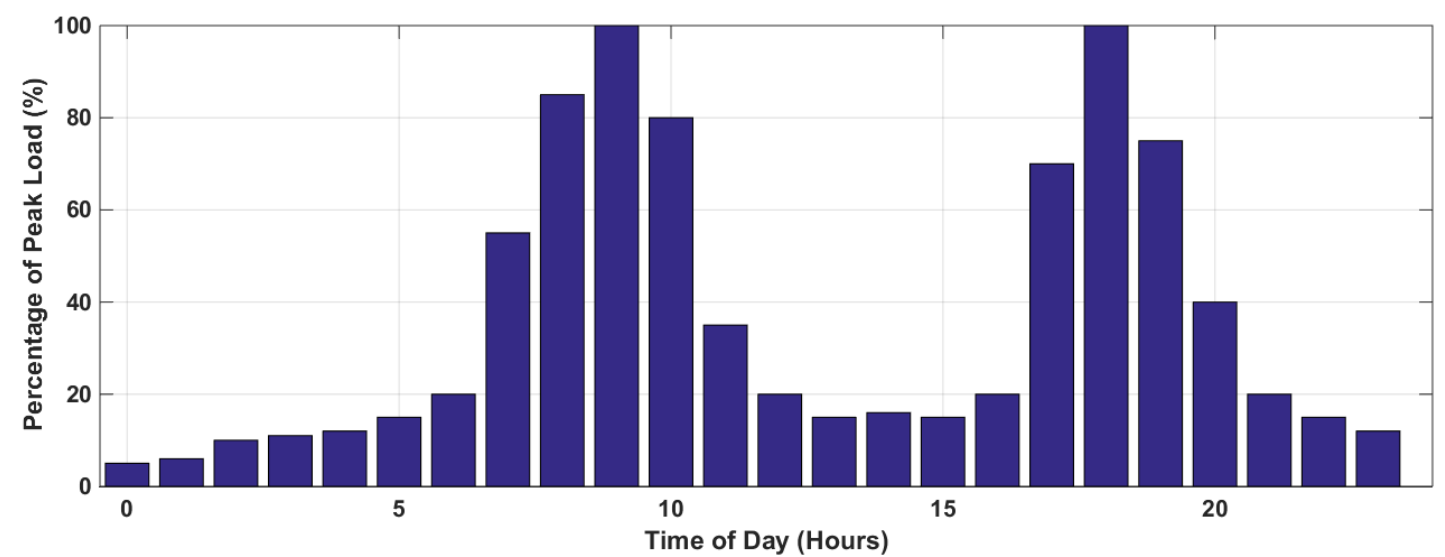

Fig. 4. Daily load curve and PEV charging activity for a charging station.

\section{PROBLEM FORMULATION}

In order to get the best optimal results, the following fitness functions will be considered. The fundamental equations for the measurement of the associated total harmonic distortions of THD $i$ current and THDV voltage are as shown by:

$$
\begin{aligned}
& \min f_{1}=T H D_{v}=\frac{\sqrt{\sum_{h=2}^{H} V_{h}^{2}}}{V_{1}} \times 100 \% \\
& \min f_{2}=T H D_{i}=\frac{\sqrt{\sum_{h=2}^{H} I_{h}^{2}}}{I_{1}} \times 100 \%
\end{aligned}
$$

$(\mathrm{H}=19$ for this article), here $\mathrm{H}$ is highest considered harmonic order.

For a m bus system, the cumulative power losses, including harmonic frequency losses, is:

$$
P_{\text {Loss }}=\sum_{h=1}^{H}\left(\sum_{i=1}^{m} P_{\text {Loss }, i}\right)
$$

Minimizing energy loss over 24-hour duration is the objective feature of the problem:

$\min f_{3}=$ Energy Loss $=\sum_{t=0}^{T} P_{\text {Loss }} \times \Delta t$ 
where at hour $\mathrm{t}$, Ploss is absolute power loss, and $\Delta \mathrm{t}$ is the interval of time (usually taken as 1 hour.

There are few conclusions and, as seen below, shortcomings would be taken into account in this article:

$$
V_{i, \text { min }} \leq V_{i r m s} \leq V_{i, \max }
$$

where the corresponding minimum and maximum rms voltage limits are $\mathrm{V}_{\mathrm{i}, \min }$ and $\mathrm{V}_{\mathrm{i}, \max }$.

Total harmonic distortion of voltage (THDv):

$$
T H D_{v} \leq T H D_{v, \max }
$$

where the distortion and the full distortion are allowed, respectively, THDv and THDv, max.

Total harmonic distortion of current (THDi):

$$
T H D_{i} \leq T H D_{i, \max }
$$

where distortion and maximum distortion are permitted, respectively, $\mathrm{THD}_{\mathrm{i}}$ and $\mathrm{THD}_{\mathrm{i}, \max }$.

(I) According to the client's request, the docking station can be plugged out / plugged in at any moment. At the period of plug-in, clients will report their plug out period requested and last SOCs requested. When SOC hits the requested SOC, the charging station is changed to stand-by mode.

(II) The time slot for one day is fifteen minutes, or equivalent to ninety six slots.

(III) The aggregator has access to data about the charging station, including its bus positions, information about plugout time, battery capacity, charger models, plug-in time, and harmonic distortion.

(IV) The charging station is controllable and has variable charging characteristics. All charging station is presumed to have a variable active load during the charging phase. The electricity used to charge the battery is dependent on the aggregator's calculations.

(V) The required time for the charging station must be more than the least charging period needed to charge device.

The optimization methodology is used in this paper to define optimized Power Active filter switching. Basically, the MOPSO method begins with random population generation before fitness estimation. To place and scale the vector filter, five parameters, which are apparent power losses, average
THDi, THDi summation, maximum THDi, and maximum THDV for the overall method, will be used. The three criteria surrounding THDi are to guarantee that the THDi is within the acceptable range on all buses.

\section{RESUlT AND DisCUSSION}

The electric car charging station is simulated and charged by a $250 \mathrm{kVA} 20 / 0.4 \mathrm{kV}$ converter, linked to grid by a $400 \mathrm{~V}$ 3 phase 4 wire system, as per seen in Figure 1. The current and voltage of $\mathrm{AC}$ bus was determined to approximate the output of control suggested. To estimate the efficacy of the proposed control, 2 cases will be simulated in order to:

- Without filtering.

- With the classic control active power filter control.

- With the proposed MOPSO-Fuzzy control active power filter control.

Simulation results are seen in Fig. 5, 6 before and after conducting MOPSO optimum switching control, demonstrating substantial changes in terms of voltage profile, actual power losses and THD. As a result, the introduction of MOPSO with adjustments every 15 minutes will decrease harmonics for 24 hours. Other than that, it is clear that MOPSO should be used with the Fuzzy logic controller to find the right solution to the overall system that offers the greatest change.

The cumulative harmonic distortion of THDV voltage and current THDi for the AC bus are seen in Fig. 7 and 8, respectively, resulting in maximum THDV being reported on the AC bus with 1.8750 percent and maximum THDi with a value of 2.6780 percent without the suggested MOPSOFuzzy control scheme. From the performance, there is a substantial reduction from 2.258004 percent to 1.74478 percent for maximum THDV and from 3.019816 percent to 2.16256 percent for maximum THDi after MOPSO gets the optimum switching series for the shunt active filter. In comparison to other solutions, a MOPSO- The Fuzzy method is then used to define solutions where the imaginable solution is the correct solution as long as one of its target functions is dominant. The Fuzzy technique can be used in Pareto to find the appropriate solutions. The solution contains THDV, THDi, and power loss in this analysis. Notice that the gross average THD value and the AC bus THD values are reduced to less than 5 percent in this scenario. Also, passive filters are not needed.

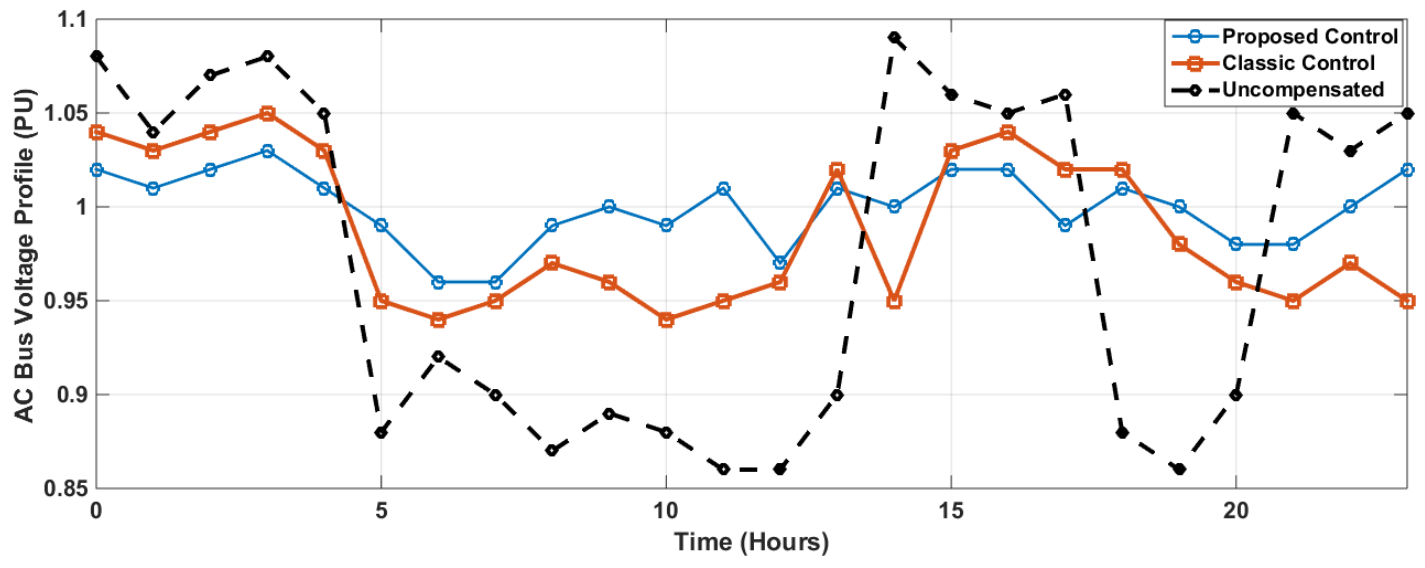

Fig. 5. Improvement of AC bus Voltage profile. 


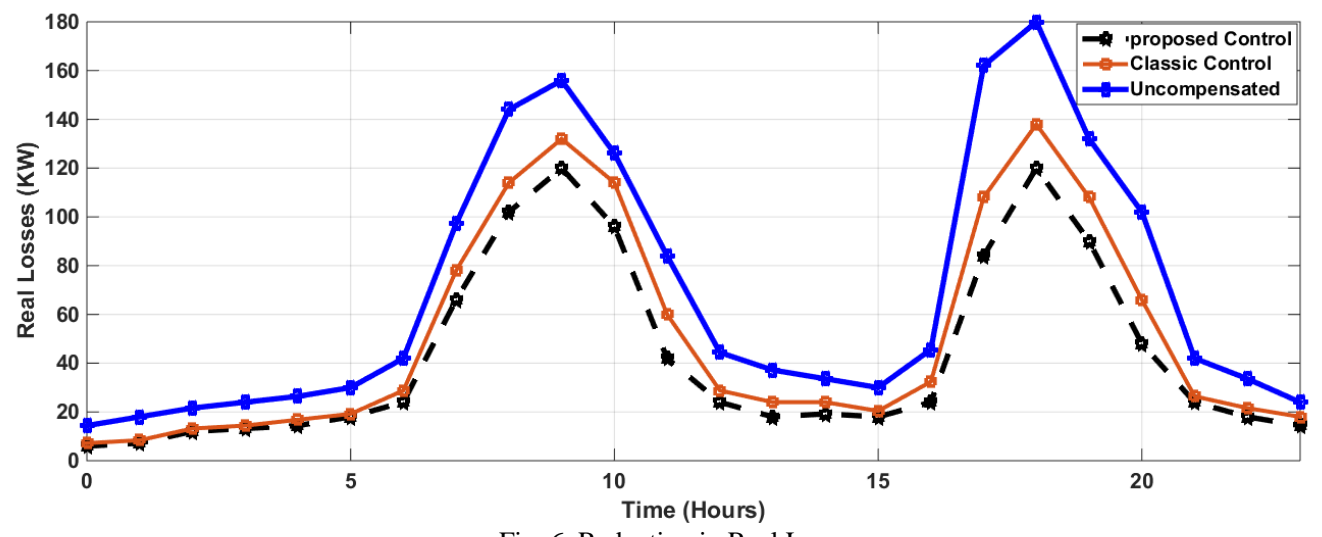

Fig. 6. Reduction in Real Losses.

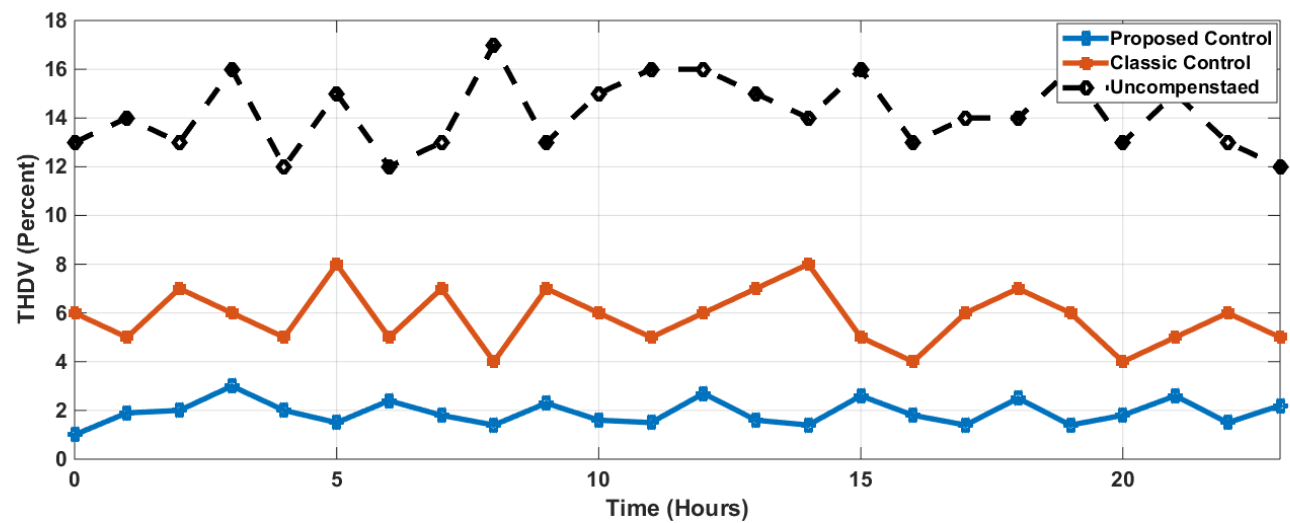

Fig. 7. Improvement of AC bus Voltage THD.

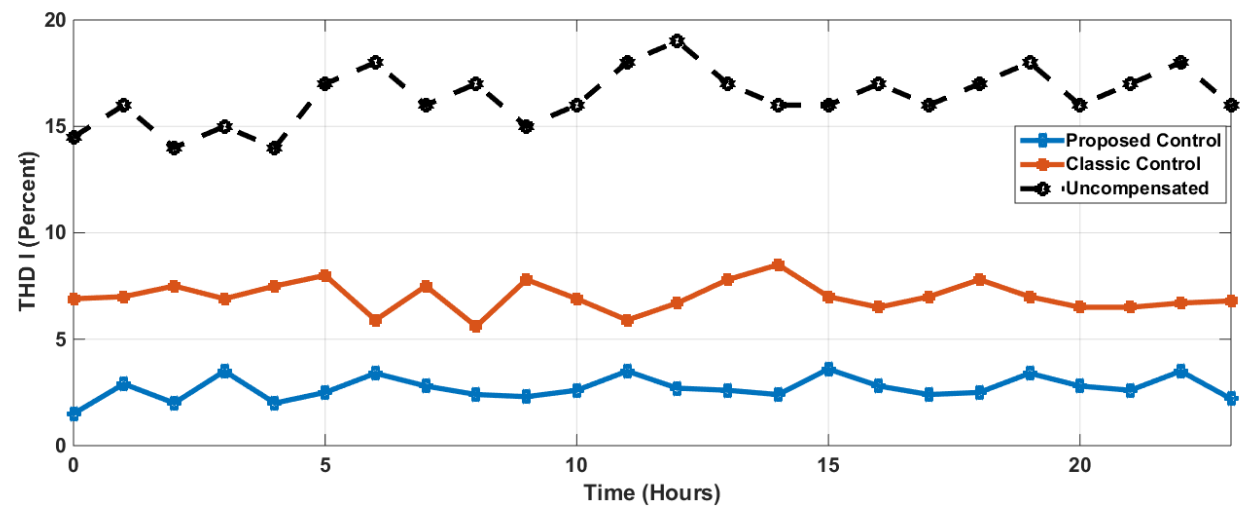

Fig. 8. Improvement of AC bus Current THD.

\section{CONCLUSION}

This paper focuses on optimum switching control of the active power filter to reduce harmonic distortion due to a delivery network PV charging station planned in 24 hours, with an interval of 15 mints depending on user behavior. To find the best switching synchronization of the active filter, MOPSO with fuzzy logic controller is used by considering the various charging station patterns and harmonic effects, which reduces the total THD for voltage and current. In the medium voltage network, thus, less harmonics are added and power losses are also minimized. The fuzzy logic controller is utilized to monitor compensation currents of shunt active power filter. It can also be assumed that MOPSO is an important instrument for harmonic reduction and helps to minimize the effects on the delivery network of the robust installation of PV charging stations. The outcomes of simulation show that proposed control scheme decreases effect of load current distortion and unbalance on the power system under balanced, non-linear and unbalanced load conditions and increases maximum THDV and maximum THDi. The additional benefits of structure are decreasing panel cost and preventing usage of power efficiency conditioner and UPS for each commercial, small business and educational institution device.

\section{REFERENCES}

[1] Mojtaba Yousefi; Amin Hajizadeh; Mohsen N. Soltani; Branislav Hredzak "Predictive Home Energy Management System with Photovoltaic Array, Heat Pump, and Plug-In Electric Vehicle" IEEE Transactions on Industrial Informatics, 2021, Volume: 17, Issue: 1.

[2] Rania A. Swief; Noha H. El-Amary; Mohamed Z. Kamh "Optimal Energy Management Integrating Plug in Hybrid Vehicle Under Load and Renewable Uncertainties" IEEE Access, 2020, Volume: 8.

[3] Sara Deilami "Online Coordination of Plugged-In Electric Vehicles and Optimal Rescheduling of Switched Shunt Capacitors in Smart Grid Considering Battery Charger Harmonics" IEEE Power and Energy Technology Systems Journal, 2018, Volume: 5, Issue: 4.

[4] Rui Wang; Qiuye Sun; Dehao Qin; Yushuai Li; Xiangke Li; Peng Wang "Steady-state Stability Assessment of AC-busbar Plug-in 
Electric Vehicle Charging Station with Photovoltaic" Journal of Modern Power Systems and Clean Energy, 2020, Volume: 8, Issue: 5.

[5] Siyang Sun; Qiang Yang; Wenjun Yan "Hierarchical optimal planning approach for plug-in electric vehicle fast charging stations based on temporal-SoC charging demand characterisation" IET Generation, Transmission \& Distribution, 2018, Volume: 12, Issue: 20.

[6] A. Lucas, F. Bonavitacola, E. Kotsakis, and G. Fulli, "Grid harmonic impact of multiple electric vehicle fast charging," Electric Power Systems Research, vol. 127, pp. 13-21, 2015.

[7] Wei Yuan; Jianwei Huang; Ying Jun Angela Zhang "Competitive Charging Station Pricing for Plug-In Electric Vehicles" IEEE Transactions on Smart Grid, 2017, Volume: 8, Issue: 2.

[8] S. Pazouki, A. Mohsenzadeh, M.-R. Haghifam, and S. Ardalan, "Simultaneous allocation of charging stations and capacitors in distribution networks improving voltage and power loss," Canadian Journal of Electrical and Computer Engineering, vol. 38, no. 2, Article ID 7097124, pp. 100-105, 2015.

[9] Li Zhai; Guixing Hu; Mengyuan Lv; Tao Zhang; Rufei Hou "Comparison of Two Design Methods of EMI Filter for High Voltage Power Supply in DC-DC Converter of Electric Vehicle" IEEE Access, 2020, Volume: 8

[10] N. Zhou, J. Wang, Q. Wang, N. Wei, and X. Lou, "Capacity calculation of shunt active power filters for electric vehicle charging stations based on harmonic parameter estimation and analytical modeling," Energies, vol. 7, no. 8, pp. 5425-5443, 2014.

[11] Jaeyeon Jo; Jinkyoo Park "Demand-Side Management with Shared Energy Storage System in Smart Grid" IEEE Transactions on Smart Grid, 2020, Volume: 11, Issue: 5.

[12] Yi Dong; Tianqiao Zhao; Zhengtao Ding "Demand-side management using a distributed initialisation-free optimisation in a smart grid" IET Renewable Power Generation, 2019, Volume: 13, Issue: 9.

[13] Huu-Cong Vu; Hong-Hee Lee "Model Predictive Current Control Scheme for Seven-Phase Voltage Source Inverter with Reduced Common-Mode Voltage and Current Harmonics" IEEE Journal of Emerging and Selected Topics in Power Electronics, 2020.

[14] Nasim Jabalameli; Xiangjing Su; Arindam Ghosh "Online Centralized Charging Coordination of PEVs With Decentralized Var Discharging for Mitigation of Voltage Unbalance" IEEE Power and Energy Technology Systems Journal, 2019, Volume: 6, Issue: 3.

[15] Julio C. Churio-Barboza; Jose Maria Maza-Ortega "Comprehensive design methodology of tuned passive filters based on a probabilistic approach" IET Generation, Transmission \& Distribution, 2014, Volume: 8, Issue: 1 .

[16] Mohit Bajaj; Amit Kumar Singh; Majed Alowaidi; Naveen Kumar Sharma; Sunil Kumar Sharma; Shailendra Mishra "Power Quality Assessment of Distorted Distribution Networks Incorporating Renewable Distributed Generation Systems Based on the Analytic Hierarchy Process" IEEE Access, 2020, Volume: 8.

[17] Soham Chakraborty; Susovan Mukhopadhyay; Sujit K Biswas "Combined Operation of D-STATCOM and Low THD SVC in a Distribution Grid for Dynamic VAr Compensation and Voltage Stabilization" 2020 IEEE International Conference on Power Electronics, Smart Grid and Renewable Energy (PESGRE2020).

[18] V. F. Corasaniti, M. B. Barbieri, P. L. Arnera, and M. I. Valla, "Hybrid power filter to enhance power quality in a medium-voltage distribution network," IEEE Trans. on Ind. Electron., vol. 56, no. 8, pp. 2885-2893, Aug. 2009.

[19] Man-Chung Wong; Ying Pang; Zeng Xiang; Lei Wang; Chi-Seng Lam "Assessment of Active and Hybrid Power Filters Under Space Vector Modulation" IEEE Transactions on Power Electronics, 2021, Volume: 36, Issue: 3.

[20] Rajasekharareddy Chilipi; Naji Alsayari; Jamal Alsawalhi "Control of dual converter-based grid-tied SPV system with series/shun compensation capabilities" IET Renewable Power Generation, 2020, Volume: 14, Issue: 1

[21] Mohammed Alhasheem; Paolo Mattavelli; Pooya Davari "Harmonics mitigation and non-ideal voltage compensation utilising active power filter based on predictive current control" IET Power Electronics, 2020, Volume: 13, Issue: 13.

[22] S. Ceballos, J. Pou, E. Robles, J. Zaragoza, and J. L. Martin, "Performance evaluation of fault-tolerant neutral point-clamped converters," IEEE Trans. Ind. Electron., vol. 57, no. 8, pp. 2709-2718, Aug. 2010.

[23] K. Vardar, E. Akpinar, and T. Surgevil, "Evaluation of reference current extraction methods for DSP implementation in active power filters," Elect. Power Syst. Res., vol. 79, pp. 1342-1352, 2009.

[24] N. Y. Dai, M. C. Wong, and Y. D. Han, "Application of a three-level NPC inverter as a three-phase four-wire power quality compensator by generalized 3 DSVM," IEEE Trans. on Power Electron., vol. 21, no. 2, pp. 440-449, Mar. 2006.
[25] H. Akagi, Y. Kanazawa, and A. Nabae, "Instantaneous reactive power compensators comprising switching devices without energy storage components," IEEE Trans. on Indl. Appl., vol. IA-20, no. 3, pp. 625$630,1984$.

[26] M. A. E. Alali, S. Saadate, Y. A. Chapuis, and F. Braun, “Advanced corrector with FPGA-based PLL to improve performance of series active filter compensating all voltage disturbances," in Proc. European Conference on Power Electron. and Appl., Aug. 2001

[27] S. Karimi, P. Poure, and S. Saadate, "High performances reference current generation for shunt active filter under distorted and unbalanced conditions," in Proc. IEEE Power Electron. Specl. Conference, Jun. 2008, pp. 195-201.

[28] M. Abdusalam, P. Poure, S. Karimi, and S. Saadate, "New digital reference current generation for shunt active power filter under distorted voltage conditions," Elect. Power Syst. Res., vol. 79, pp. 759 $765,2009$.

[29] A. Hamadi, K. Al-haddad, S. Rahmani, and H. Kanaan, "Comparison of fuzzy logic and proportional integral controller of voltage source active filter compensating current harmonics and power factor," in Proc. IEEE Int. Conference Ind. Techn., IEEE, 2004, vol. 2 , pp. 645 650.

[30] A. Dellaquila, A. Lecci, and V. G. Monopoli, "Fuzzy controlled active filter driven by an innovative current reference for cost reduction," in Proc. IEEE Int. Symp. Ind. Electron., 2002, vol. 3, pp. 948-952.

[31] M. Alonso, H. Amaris, J. G. Germain, and J.M. Galan, "Optimal charging scheduling of electric vehicles in smart grids by heuristic algorithms," Energies, vol. 7, no. 4, pp. 2449-2475, 2014.

[32] G. R. Bharati and S. Paudyal, "Coordinated control of distribution grid and electric vehicle loads," Electric Power Systems Research, vol. 140 pp. 761-768, 2016

[33] M. A. S. Masoumand S. M. H. Nabavi, "Hybrid optimal online overnight charging coordination of plug-in electric vehicles in smart grid," Journal of Power Sources, vol. 330, pp. 7-17, 2016 